\title{
Nucleation of Slip Bands near Twin Boundary in High-Cycle Fatigue*
}

\author{
Takashi SUMIGAWA ${ }^{* * * * *}$ and Takayuki KITAMURA***
}

\begin{abstract}
In order to investigate the criterion for the persistent slip band (PSB) formation near a $\Sigma 3(111)$ coherent twin boundary, where is a preferential site for formation of PSB, a highcycle fatigue test is carried out at room temperature using a copper bicrystal specimen with the twin boundary. PSBs are observed near the boundary in the connection with the evolution of extrusion/intrusion on the specimen surface. The slip behavior is different from that predicted by the Schmid factor. A finite element method (FEM) analysis is conducted for the bicrystal, and it reveals that the increase of resolved shear stress on the specific slip system due to the constraint of deformation between the crystals is the main cause for promoting the nucleation of PSB near the twin boundary. By taking into account the interaction between the primary and the secondary slip systems, it is possible to specify the formation behavior of PSB near the twin boundary.
\end{abstract}

Key Words: Fatigue, Stress Concentration, Finite Element Method, $23(111)$ Twin Boundary, Persistent Slip Band, Bicrystal, Constraint

\section{Introduction}

The formation of persistent slip band (PSB) is an essential phenomenon of local deformation in the early stage of fatigue of metals ${ }^{(1),(2)}$. A grain boundary, which is an interface between crystals in a polycrystal, has a great influence on the formation of PSB $^{(3)-(6)}$. It is well known that the PSB is easy to be formed near a coherent twin boundary ( $\Sigma 3(111)$ boundary) in high-cycle fatigue of face centered cubic metals and it is a preferential site for the crack initiation $^{(7)-(11)}$. Thus, it is weak point in highcycle fatigue though the twin boundary possesses high endurance for stress corrosion cracking ${ }^{(12)}$ or grain boundary embrittlement in liquid metal ${ }^{(13)}$.

In this study, in order to investigate the criterion of PSB formation near the twin boundary, a high-cycle fatigue test and a finite element method (FEM) analysis are carried out for a bicrystal specimen with the twin bound-

* Received 16th June, 2003 (No. 01-1282). Japanese Original: Trans. Jpn. Soc. Mech. Eng., Vol.68, No.671, A(2002), pp.1104-1111 (Received 19th October, 2001)

** Mechanical Engineering Research Laboratory, Hitachi Ltd., Tsuchiura, Ibaraki 300-0013, Japan. E-mail: sumigawa@gm.merl.hitachi.co.jp

*** Department of Engineering Physics and Mechanics, Kyoto University, Yoshidahommachi Sakyo-ku, Kyoto 606-8501, Japan ary.

\section{Experimental Procedure}

\subsection{Bicrystal specimen}

The crystal orientation of each grain in a plate of copper polycrystal ( $99.9999 \%$ mass) is examined by means of an orientation imaging microscope (OIM). After a $\Sigma 3(111)$ boundary (coherent twin boundary) is specified, a bicrystal specimen with the twin boundary, which tilts to the stress (longitudinal) direction, is extracted using an electric-discharge cutting machine. It should be noted that the specimen consists of only two crystals (Fig. 1) where the upper and the lower ones are named as "Crystal 1" and "Crystal 2", respectively. The specimen is annealed cyclically for homogenization at $973 \mathrm{~K} 0.5 \mathrm{~h}+$ $1073 \mathrm{~K} 0.5 \mathrm{~h}$ for $48 \mathrm{~h}$ in a vacuum after mechanical polishing. The surface is polished by buffing and is electrically finished up in phosphoric acid-ethanol mixture. The shape and dimension of the specimen and the stereographic projection of each crystal are shown in Fig. 1 . The twin boundary possesses the angle of $54.6^{\circ}$ to the stress axis. Two side surfaces, on which a detailed surface observation is performed, are designated as "obverse surface" and "reverse surface", respectively (See Fig. 1).

\subsection{Crystallographic characteristics of specimen}

The orientations of the component crystals are symmetrical on the twin boundary ((111) plane), and their 


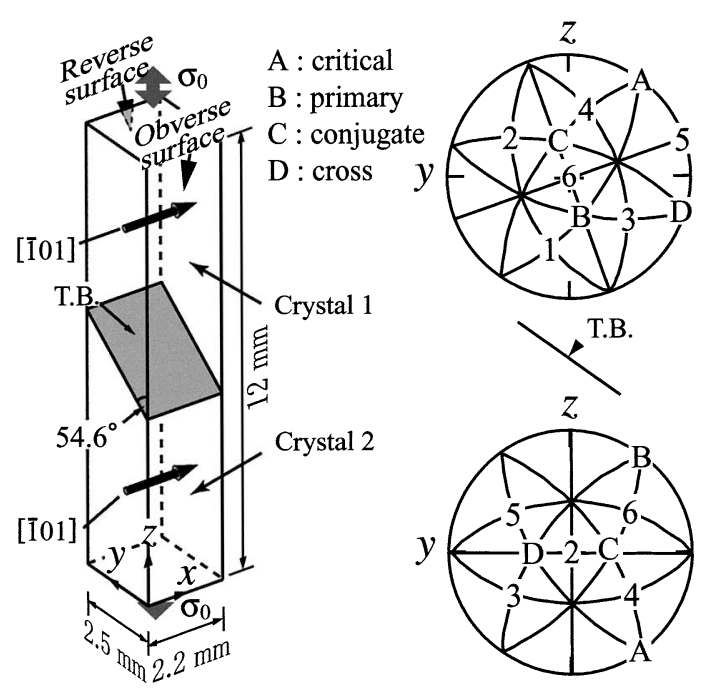

Fig. 1 Shape and size of bicrystal specimen, and stereographic projections of the component crystals
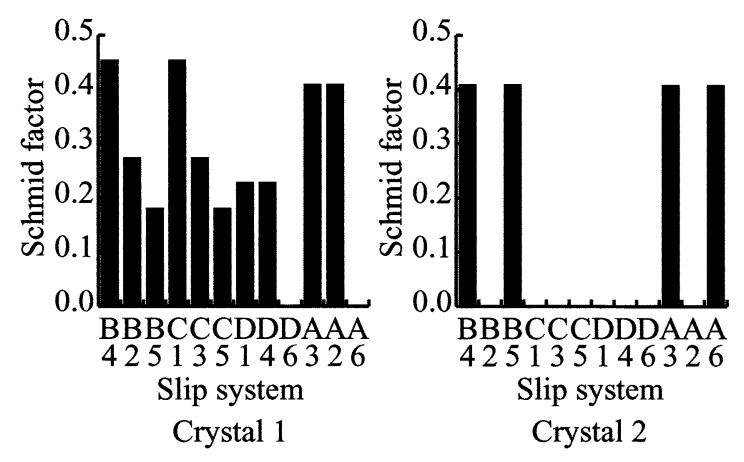

Fig. 2 Schmid factors of 12 slip systems in the component crystals

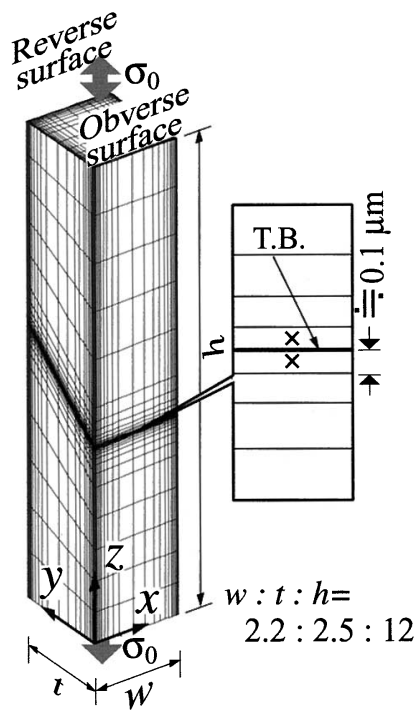

(a) FEM mesh of the bicrystal

Crystal 2

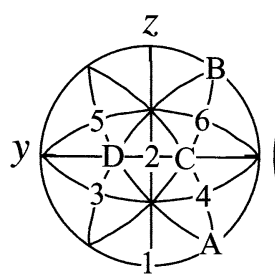

$\phi=0^{\circ}$

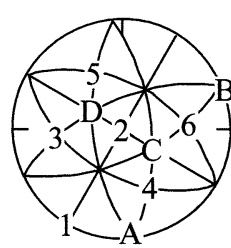

$\phi=30^{\circ}$

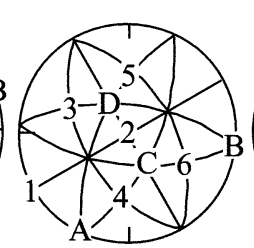

$\phi=60^{\circ}$

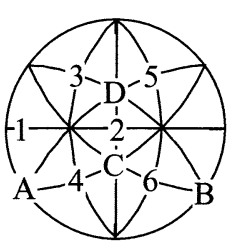

$\phi=90^{\circ}$

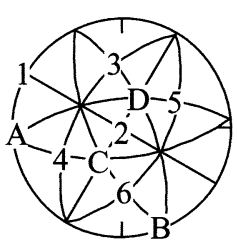

$\phi=120^{\circ}$

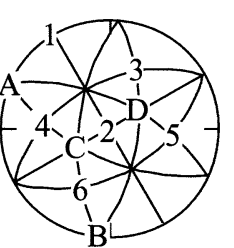

$\phi=150^{\circ}$

(b) Stereographic projections of rotated Crystal 2

Fig. 3 Finite element model for stress analysis 
[101] directions are parallel as shown by the arrows in Fig. 1. In each stereographic projection, the four slip planes and the six slip directions are termed as A-D and $1-6$ by the Schmid and Boas notation, respectively. The primary slip system is represented as B4 by the notation. The Schmid factors of 12 slip systems in both crystals are shown in Fig. 2. In Crystal 1, the Schmid factors on B4 and $\mathrm{C} 1$ are equal and the largest. In Crystal 2, the Schmid factors on B4, B5, A3 and A6 are almost equal and the largest. These Schmid factors suggest that multiple slip systems are activated simultaneously in each crystal. In fatigue of copper single crystal with multiple slip orientation, it is reported that only one slip system activates mainly after the initial interaction among the dislocations, and PSB is formed on the slip system ${ }^{(14),(15)}$.

\subsection{High cyclic fatigue test}

A pull-push cyclic deformation test is carried out using a servo-hydraulic fatigue-testing machine. So as to avoid the initial strain introduced at the setting, the bottom of the specimen is inserted into a pot with melted wood metal after the top is attached to the upper jig, and it is fixed by cooling. The experiment is conducted in an air at room temperature under a triangular load waveform with the frequency of $1 \mathrm{~Hz}$. Small load is provided to the specimen at the beginning, and the amplitude is increased gradually with checking the sufficient hardening on the hysteresis loop. The obverse surface and the reverse surface are carefully examined in situ by means of a Nomarski differential interferpherometer. The experiment is terminated when the formation of PSB is observed on the specimen surface. After the fatigue test, the specimen surface is inspected in detail by means of a Nomarski differential interference microscope and a scanning electron microscope (SEM).

\section{Analytical Procedure}

MSC/NASTRAN for Windows (Version 4.6) is used for a FEM analysis. The mesh division is shown in Fig. 3 (a) where 36450 hexahedron elements are used. The aspect ratio of model size is $2.2: 2.5: 12$, which is same as the specimen used in the experiment. The regions near the twin boundary and near the specimen corner, where the stress may concentrate, are divided into fine elements so as to get accurate stress distribution. The width of element adjacent to the twin boundary is set to be about $0.1 \mu \mathrm{m}$. The elasticity of cubic crystals possesses orthotropism because of the structural symmetry, and the stress-strain relationship is represented by three elastic constants for the crystallographic axes, $C_{11}, C_{12}$ and $C_{44}$. In the analysis, we adopt the elastic constants $C_{11}=168.4 \mathrm{GPa}, C_{12}=75.4 \mathrm{GPa}$ and $C_{44}=121.4 \mathrm{GPa}^{(16)}$ for each crystal taking into account their orientations. The uniform normal stress, $\sigma_{0}$, is applied to the top and the bottom.
The stress distributions in bicrystals with different Crystal 2 orientation are also analyzed. When the orientation of Crystal 2 rotates $180^{\circ}$ for the $x$ axis, the atomic arrangement perfectly corresponds with the original one because the $x$ axis parallel to the [101] direction is a crystallographic two-fold axis. Thus, the six models, where Crystal 2 is rotated by $0^{\circ}$ (twin boundary), $30^{\circ}, 60^{\circ}, 90^{\circ}$, $120^{\circ}$ and $150^{\circ}$, are adopted in the analysis for comparison (Fig. 3 (b)).

\section{Results and Discussions}

\subsection{Surface observation}

The specimen surface has no striking change up to the stress amplitude of $35 \mathrm{MPa}$ (100000 cycles). Then, the thick slip bands are observed near the twin boundary on the reverse surface at the stress amplitude of $44 \mathrm{MPa}$ (128 000 cycles), and the test is terminated. The largest Schmid factors in Crystal 1 and Crystal 2 are 0.454 (B4, C1) and 0.41 (B4, B5, A3, A6), respectively. The maximum resolved shear stress amplitudes in each crystal, which are calculated by the largest Schmid factors and the applied stress amplitude $(\Delta \sigma / 2=44 \mathrm{MPa})$ are $\Delta \sigma / 2$. $0.454 \fallingdotseq 20.0 \mathrm{MPa}($ Crystal 1$)$ and $\Delta \sigma / 2 \cdot 0.41 \fallingdotseq 18.0 \mathrm{MPa}$ (Crystal 2), respectively.

Figure 4 shows micrographs near the twin boundary on the surfaces. Thick slip bands are clearly identified near the twin boundary on the reverse surface while no slip band is found on the obverse surface. The slip bands must be PSBs because characteristic extrusions/intrusions

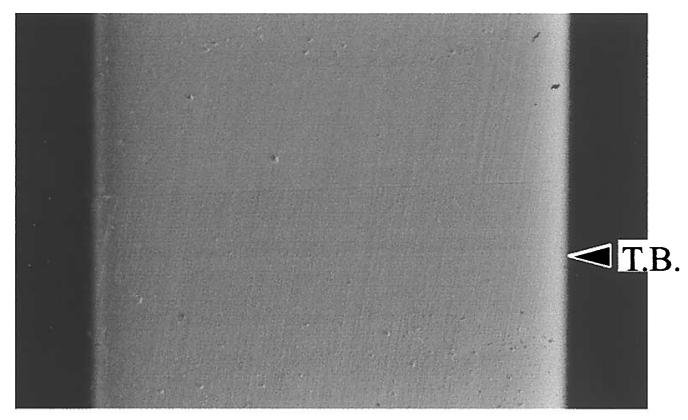

Obverse surface

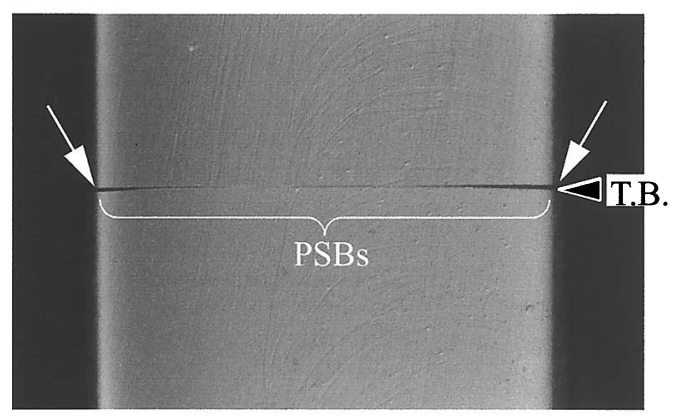

Reverse surface

Fig. 4 Photographs of slip patterns near the twin boundary $(N=$ $1.28 \times 10^{5}$ cycles, $\left.\Delta \sigma / 2=44 \mathrm{MPa}\right)$ 


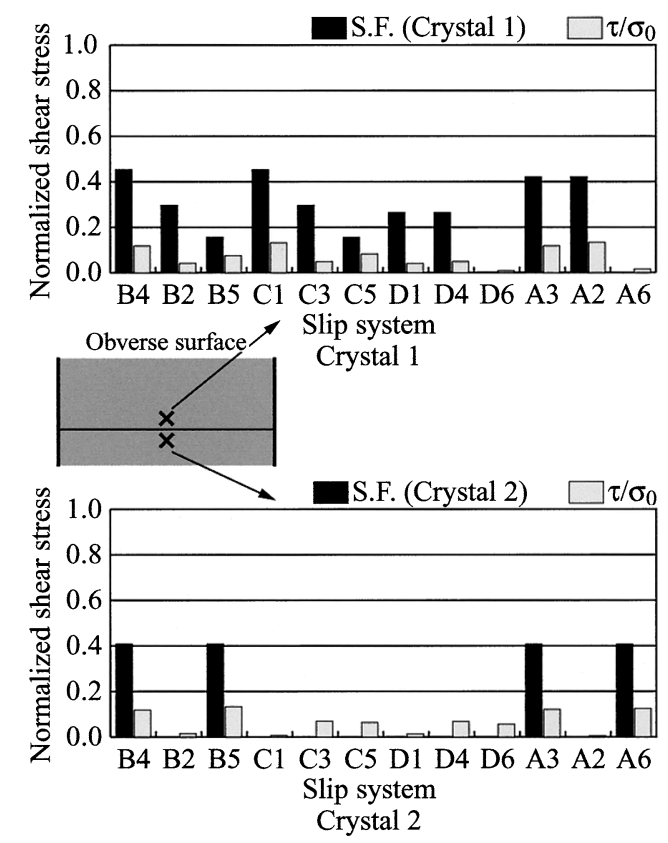

(a) Obverse surface

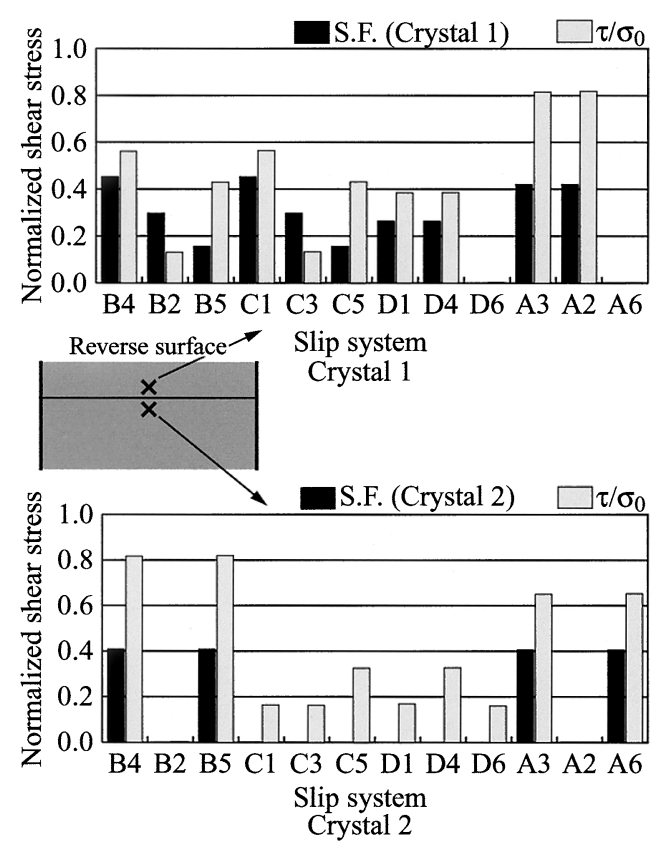

(b) Reverse surface

Fig. 5 Schmid factors (SFs) and magnitudes of $\tau / \sigma_{0}$ on the 12 slip systems near the twin boundary

are found in the bands. A paper, which reports preferential formation of PSB near a twin boundary ${ }^{(7)}$ supports it. On the reverse surface, the width of the PSBs is not uniform along the twin boundary, and it widen near the specimen corner (arrows in the figure). The crystal orientation signifies that the PSBs are formed on the slip plane A in Crystal 1 and on the slip plane B in Crystal 2, respectively.

\subsection{Shear stress on slip system}

Figure 5 shows the normalized resolved shear stress on each slip system, $\tau / \sigma_{0}$, in the elements adjacent to the twin boundary at the center of the obverse surface and the reverse surface (" $X$ " points in Fig. 3 (a)). In the figure, the Schmid factors in each crystal are also plotted for comparison. The Schmid factor on each slip system is constant everywhere in the crystal because it is dependent on only the crystal orientation and the loading direction. The Schmid factor represents the normalized resolved shear stress at the region away from the twin boundary without the influence of neighboring crystal. On the other hand, $\tau / \sigma_{0}$ represents the stress influenced by the constraint of neighboring crystal at the twin boundary.

On the obverse surface (Fig. 5 (a)), the magnitudes of $\tau / \sigma_{0}$ on all slip systems are small in both crystals. As the resolved shear stress on all slip system decreases near the twin boundary, the crystallographic slip is suppressed. Thus, the PSB is not formed on the obverse surface.

On the reverse surface (Fig. 5 (b)), the magnitudes of $\tau / \sigma_{0}$ on A 3 and A 2 in Crystal 1 and on B4 and B5 in Crystal 2 are much larger than the largest Schmid factor in each crystal. The slip plane A in Crystal 1 and the slip plane B in Crystal 2 are parallel to the twin boundary. It suggests that the PSBs along the twin boundary on the reverse surface are brought about by the increase of the shear stress on the slip systems parallel to the twin boundary.

\subsection{Condition of PSB formation}

4.3.1 Effect of specimen corner Figure 6 shows SEM photographs at the twin boundary near the specimen corner $\left(x^{\prime}=1.05 \mathrm{~mm}\right)$ and at the center of the twin boundary $\left(x^{\prime}=0 \mathrm{~mm}\right)$. Near the specimen corner (Fig. 6(a)), the extrusions are formed within about $5.5 \mu \mathrm{m}$ from the boundary in Crystal 1 and about $10 \mu \mathrm{m}$ in Crystal 2. The slips are remarkably activated with approaching the twin boundary. At the center (Fig. 6(b)), the extrusion width is about $1 \mu \mathrm{m}$. The crystal orientations indicate that the PSBs are formed by the activation of A3 or A2 in Crystal 1 and B4 or B5 in Crystal 2, respectively ${ }^{(17)}$.

$x^{\prime}$ axis, of which origin is set at the center, is defined along the twin boundary (See Fig. 6). Figure 7 shows the changes of $\tau / \sigma_{0}$ along the $x^{\prime}$ axis. The broken lines $\left(x^{\prime}=0 \mathrm{~mm}, x^{\prime}=1.05 \mathrm{~mm}\right)$ in the figure indicate the points where the SEM photographs are taken.

In Crystal $1, \tau / \sigma_{0}$ on $\mathrm{A} 2$ is the largest in the left side while it on A3 is the largest in the right side. Near the center of the twin boundary, $\tau / \sigma_{0}$ on A 3 and $\mathrm{A} 2$ are almost equal, so that "coplanar slip", in which two identical slip systems on a slip plane are active simultaneously, takes place.

In Crystal 2, $\tau / \sigma_{0}$ on B5 is the largest in the left side while it on B4 is the largest in the right side. However, $\tau / \sigma_{0}$ on B4 and B5 are almost equal near the center, which 

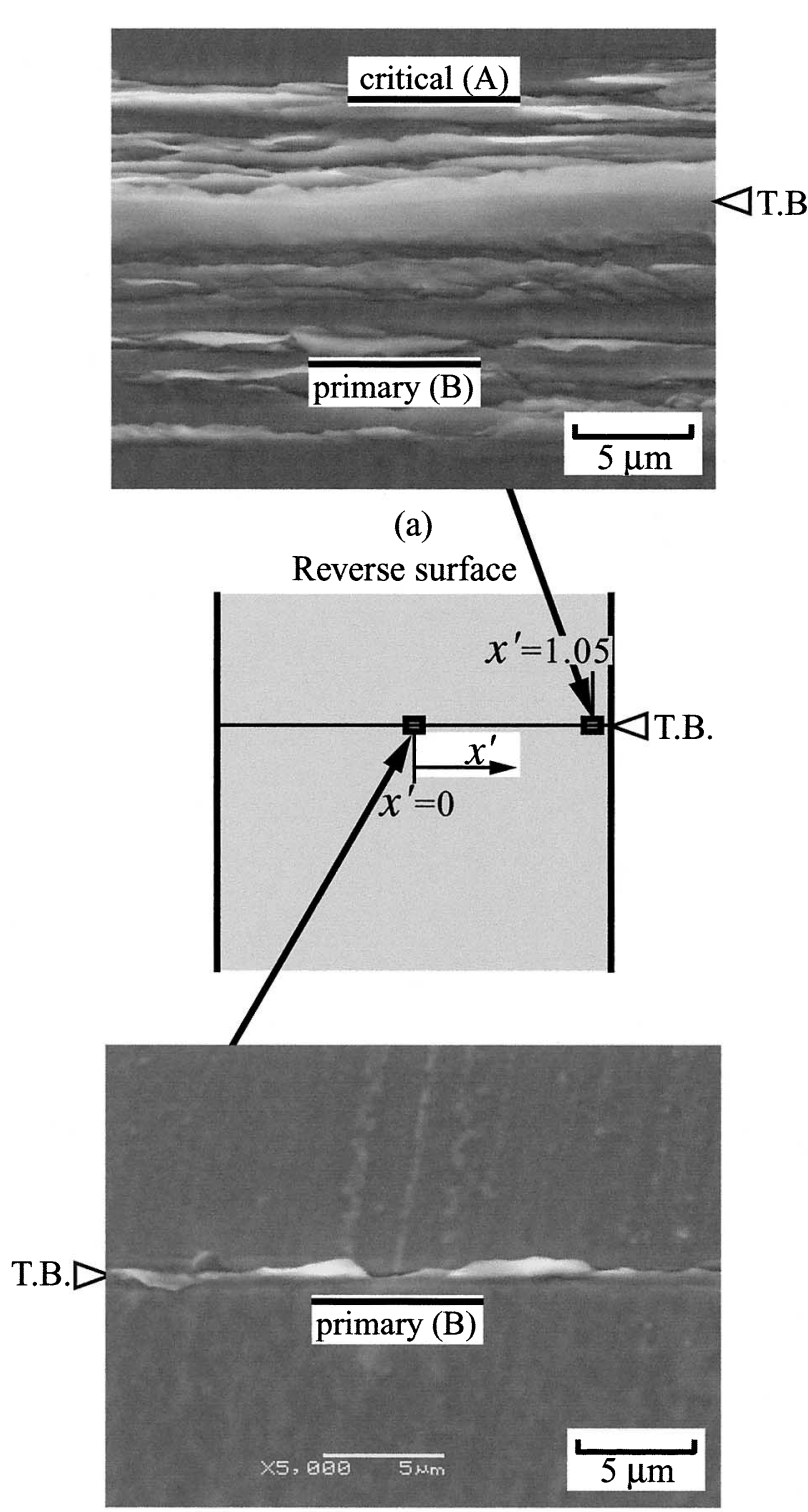

(b)

Fig. 6 SEM photographs of PSB morphology near the twin boundary on Reverse surface (a) near the specimen edge and (b) at the center $\left(N=1.28 \times 10^{5}\right.$ cycles, $\Delta \sigma / 2=$ $44 \mathrm{MPa})$

brings about the coplanar slip.

\subsubsection{Near specimen corner on the twin boundary}

The activation of secondary slip system has great influence on the dislocation structure of PSB. The difference of the extrusions width between the specimen corner and the center may be attributed to such difference due to the stress conditions. The changes of $\tau / \sigma_{0}$ on the specific slip systems near the specimen corner with the distance from the twin boundary at the reverse surface $\left(x^{\prime}=1.05 \mathrm{~mm}, x_{1}^{\prime}\right.$ and $x_{2}^{\prime}$ axes : Fig. 8) are shown in Fig. 9. While $\tau / \sigma_{0}$ at the region away from the twin boundary are almost equal to the Schmid factors in both crystals, they become larger near the boundary. In Crystal 1 (Fig. 9(a)), eminent in-
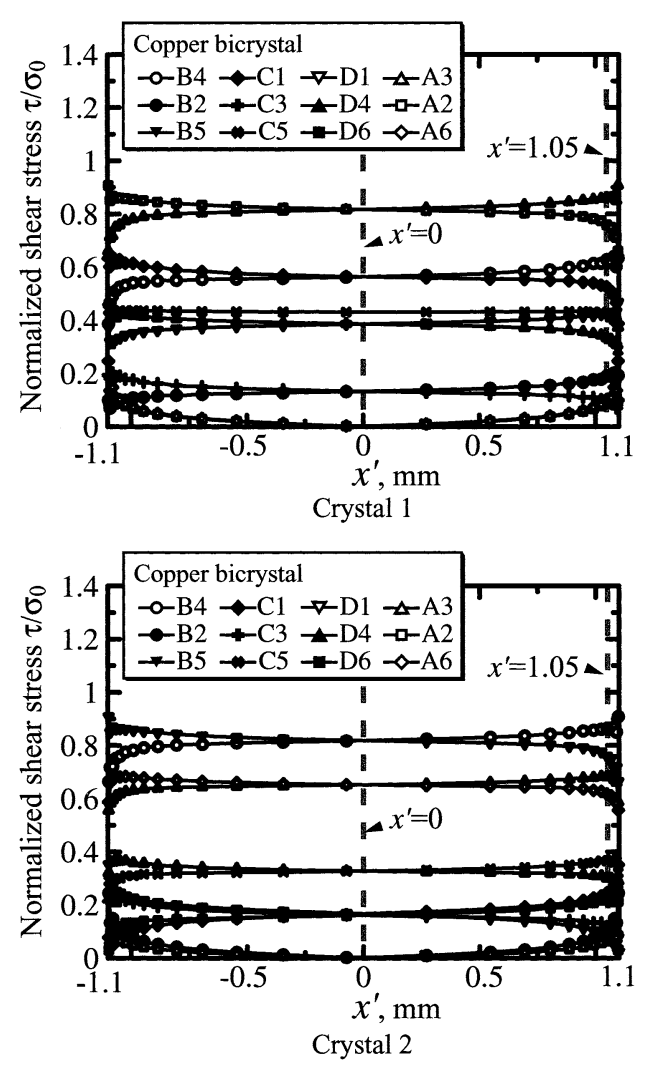

Fig. 7 Distribution of $\tau / \sigma_{0}$ along the twin boundary on Reverse surface

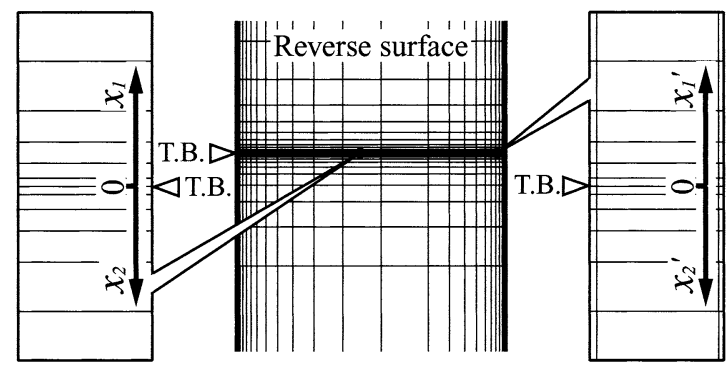

Fig. 8 Elements where $\tau / \sigma_{0}$ is extracted.

crease of $\tau / \sigma_{0}$ on $\mathrm{A} 3$ is recognized at the region where the extrusions are formed $\left(x_{1}^{\prime}=5.5 \mu \mathrm{m}\right)$. Thus, the criterion for PSB formation is determined as 0.68 from $\tau / \sigma_{0}$ at $x_{1}^{\prime}=5.5 \mu \mathrm{m}$. As the maximum nominal stress amplitude is $\Delta \sigma / 2 \fallingdotseq 44 \mathrm{MPa}$, the critical resolved shear stress amplitude is $\Delta \sigma / 2 \cdot 0.68 \fallingdotseq 29.9 \mathrm{MPa}$. This agrees with the PSB formation stress of a copper single crystal $(\fallingdotseq 28.0$ $\mathrm{MPa})^{(18)}$.

In Crystal 2 (Fig. 9 (b)), $\tau / \sigma_{0}$ on B4 increases at the region within about $300 \mu \mathrm{m}$ from the twin boundary. The critical $\tau / \sigma_{0}$ on B4 at the point where the extrusions start to generate $\left(x_{2}^{\prime}=10 \mu \mathrm{m}\right)$ is about 0.68 . The resolved shear stress amplitude is $29.9 \mathrm{MPa}$, and agrees with the PSB formation stress in a copper single crystal as well.

These results support that the PSB formation in the single slip near the twin boundary is governed by both 


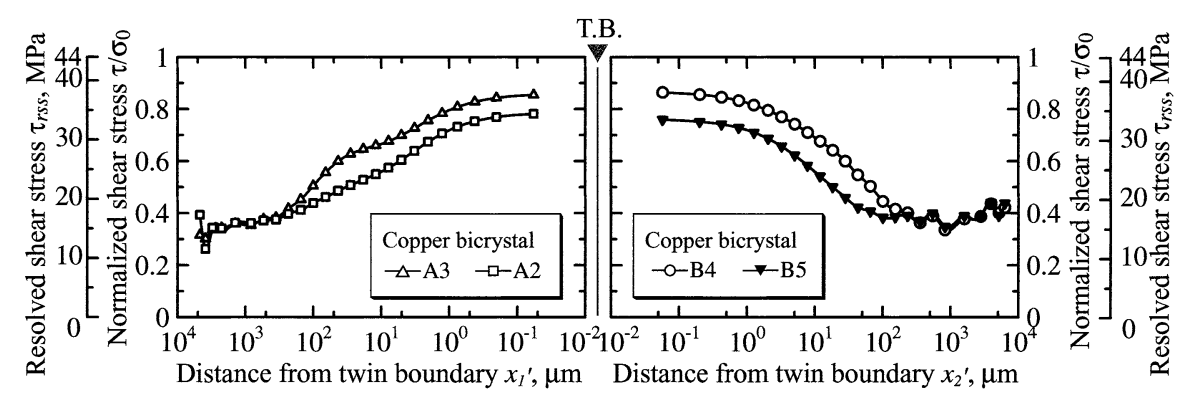

(a) Crystal 1

(b) Crystal 2

Fig. 9 Change in $\tau / \sigma_{0}$ from the twin boundary near the specimen edge

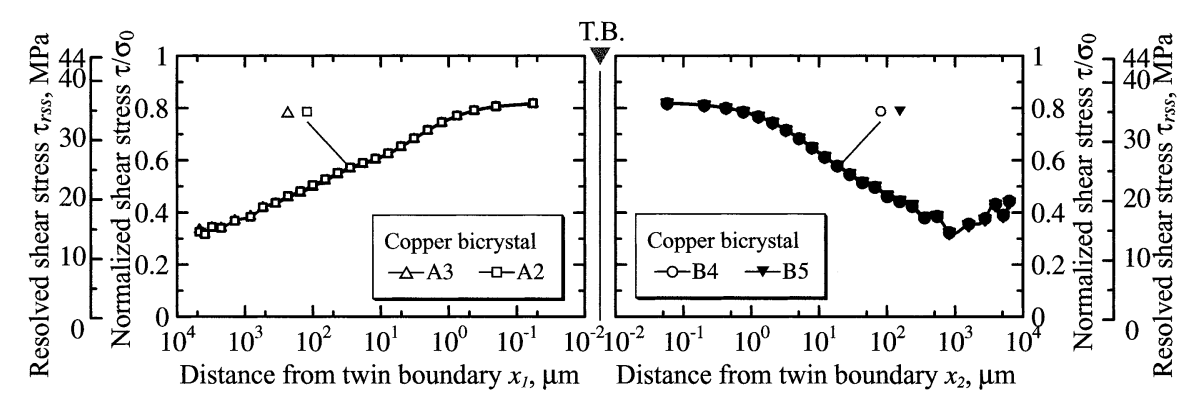

(a) Crystal 1

(b) Crystal 2

Fig. 10 Change in $\tau / \sigma_{0}$ from the twin boundary at the center

the maximum resolved shear stress and the PSB formation stress of copper single crystal.

\subsubsection{Center of twin boundary Figure 10} shows the changes in $\tau / \sigma_{0}$ on the specific slip systems with the distance from the twin boundary in each crystal at the center of the reverse surface $\left(x^{\prime}=0 \mathrm{~mm}, x_{1}\right.$ and $x_{2}$ axes : Fig. 8). $\tau / \sigma_{0}$ at the region away from the twin boundary are almost equal to the Schmid factors.

In Crystal 1 (Fig. $10(\mathrm{a})$ ), there is no difference between the magnitudes of $\tau / \sigma_{0}$ on A3 and A2, and they increase near the twin boundary. The extrusions are formed within the width of about $1 \mu \mathrm{m}$. Assuming that the extrusion widths are $0.5 \mu \mathrm{m}$ in both crystals, the critical $\tau / \sigma_{0}$ for the PSB formation is $0.79\left(x_{1}=0.5 \mu \mathrm{m}\right)$, which is the resolved shear stress amplitude of $\Delta \sigma / 2 \cdot 0.79=34.8 \mathrm{MPa}$.

In Crystal 2 (Fig. 10 (b)), there is no difference between the magnitudes of $\tau / \sigma_{0}$ on B4 and B5, and they increase near the twin boundary. The critical $\tau / \sigma_{0}$ on B4 (B5) is $0.8\left(x_{2}=0.5 \mu \mathrm{m}\right)$, which is the resolved shear stress amplitude of $\Delta \sigma / 2 \cdot 0.8=35.2 \mathrm{MPa}$.

It can be concluded that the extrusion formation stress in coplanar slip is about $35.0 \mathrm{MPa}$, which is larger than the PSB formation stress of copper single crystal with single slip orientation $(=28.0 \mathrm{MPa})$. In a copper single crystal with coplanar slip orientation, the dislocations on the active slip plane form cell structure and the fine extrusions are formed on the surface ${ }^{(14)}$. Although the critical stress for the extrusion formation in copper single crystal with coplanar slip orientation is not clarified yet, a report sug- gested that the formation is dependent on the peak shear stress amplitude. It is about $\tau_{\text {sat. }}=36 \mathrm{MPa}^{(14)}$ for copper and it agrees with the extrusion formation stress obtained in this study.

After all, the local stress field owing to the deformation constraint of the neighboring crystal governs the formation of specific PSB near the twin boundary in highcycle fatigue. And, it is possible to describe the PSB formation behavior in view of the dislocation interaction between the primary and the secondary slip systems controlled by the magnitude of the resolved shear stress.

\subsubsection{Effect of crystal orientation on shear stress} near grain boundary The effect of Crystal 2 orientation on the local stress is examined by the FEM. Figure 11 shows the $\tau / \sigma_{0}$ on 12 slip systems in the elements at the center of the boundary where Crystal 2 is rotated around the $x$ axis by $30^{\circ}$.

On the obverse surface, the magnitudes of $\tau / \sigma_{0}$ in the models are slightly dependent on $\phi$ and are smaller than 0.39 .

On the reverse surface, $\tau / \sigma_{0}$ on a slip system exceeds 0.8 at $\phi=0^{\circ}$ (twin boundary) and $\phi=120^{\circ}$. Thus, the twin boundary gives the highest stress among the models. This does not mean that the twin boundary is the most favorable site for the PSB formation because $\tau / \sigma_{0}$ is affected by not only crystal orientation of the neighboring crystal (grain boundary structure) but also the crystal shape and the loading direction. However, the high resolved shear stress on the slip plane parallel to the twin boundary (con- 

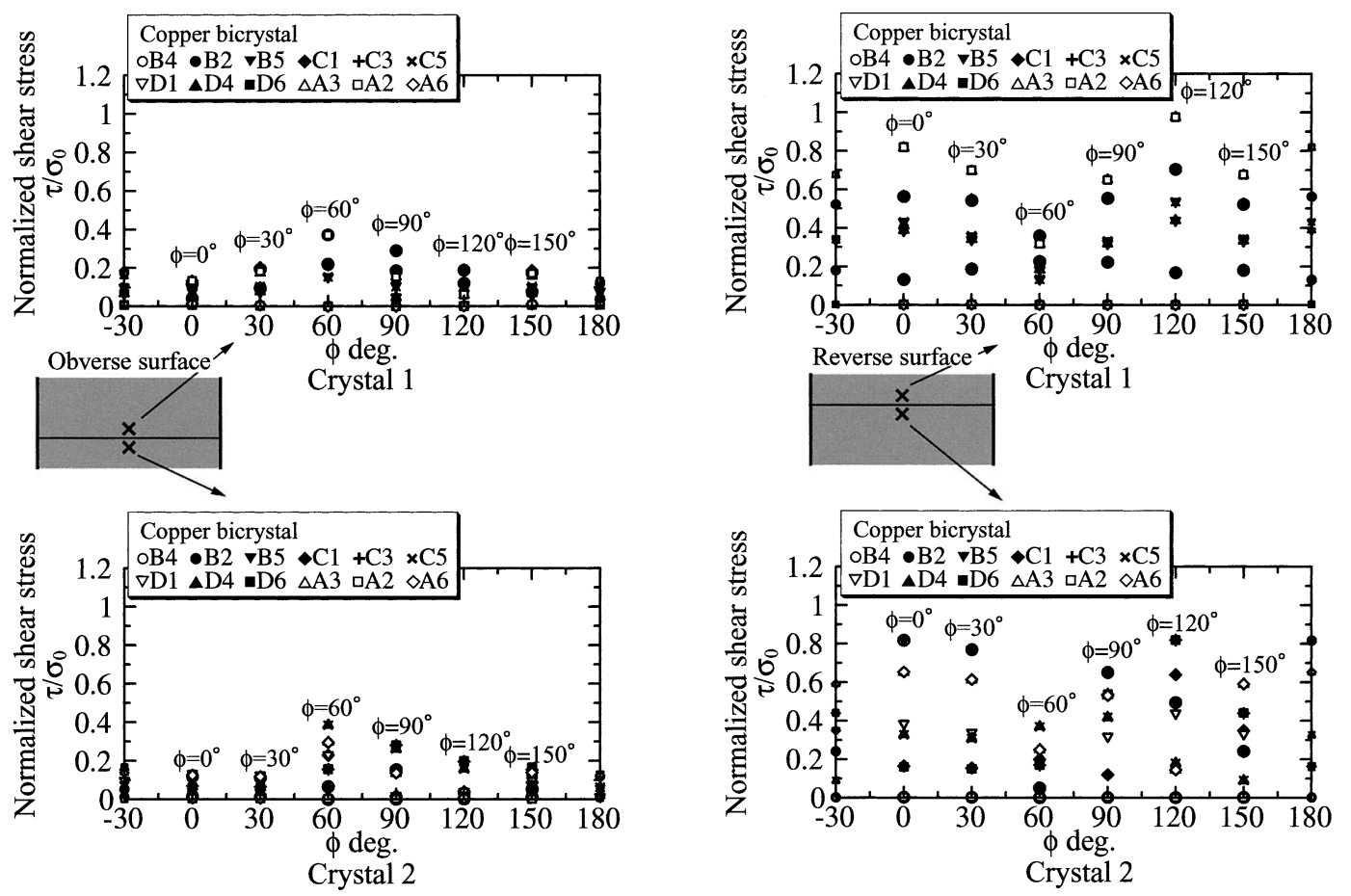

(a) Obverse surface

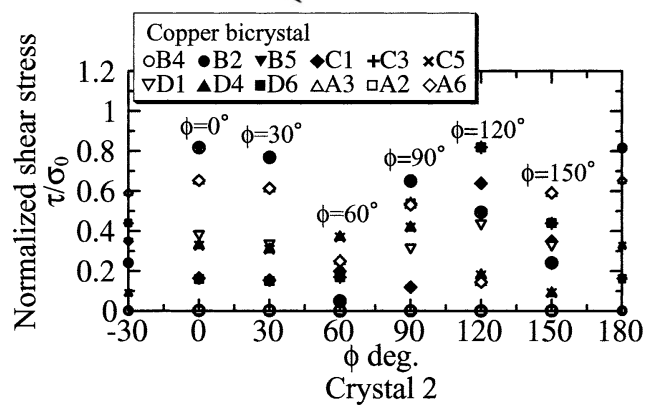

(b) Reverse surface

Fig. 11 Effect of rotated Crystal 2 on the normalized shear stress, $\tau / \sigma_{0}$, in elements at the center of the boundary

straint boundary) is a reasonable explanation for the cause of PSB formation near the twin boundary.

\section{Conclusion}

A high-cycle fatigue test is carried out using a bicrystal specimen with a twin boundary tilted to the loading direction. The detailed surface observation gives the following results.

1. PSBs that are parallel to the twin boundary are formed near the boundary.

2. The morphology of PSBs changes along the twin boundary.

3. The slip behavior near the twin boundary is different from that predicted by the Schmid factor.

A stress analysis of the specimen by FEM gives the following results.

4. Stress concentrates at the region near the twin boundary due to the deformation constraint of the neighboring crystal.

5. High magnitude of $\tau / \sigma_{0}$ is observed on the activated slip system where the PSBs are formed.

6. The formation behavior of PSB near the twin boundary can be described by the magnitude of the resolved shear stress considering the dislocation interaction.

7. The formation of specific PSB near the twin boundary is governed by the formation stress of single crystal.
8. Analysis for the models with different orientation of Crystal 2 reveals that there are slip systems with high resolved shear stress near the twin boundary. Their stresses are larger than those of slip systems near the other boundaries.

\section{References}

( 1 ) Thompson, N., Wadsworth, N. and Louat, N., The Origin of Fatigue Fracture in Copper, Phil. Mag., Vol.1 (1956), pp.113-126.

( 2 ) Essmann, U., Gosele, U. and Mughrabi, H., A Model of Extrusions and Intrusions in Fatigued Metals I. PointDefect Production and the Growth of Extrusions, Phil. Mag. A, Vol.44, No.2 (1981), pp.405-426.

( 3 ) Sittner, P., Novak, V. and Braidler, J., Persistent Slip Band-Grain Boundary Interactions in Low Strain Fatigue of Isoaxial Fe-14wt.\%Cr Bicrystals Scri. Metall. Mater., Vol.27 (1992), pp.705-710.

( 4 ) Sumigawa, T., Wada, T., Hashimoto, S. and Kitamura, T., Analysis of Slip Behavior in Two-phase $(\alpha / \beta)$ Stainless Bicrystal with Longitudinal Interface in High Cycle Fatigue, Transactions of JSME, (in Japanese), Vol.67, No.656 (2001), pp.732-739.

( 5 ) Kitamura, T., Sumigawa, T. and Ohishi, K., Slip Behavior and Local Stress near Grain Boundary in HighCycle Fatigue of Multi-Crystalline Copper, Transactions of JSME, (in Japanese), Vol.67, No.663 (2001), pp.1819-1824.

( 6 ) Kitamura, T., Sumigawa, T., Wada, T. and Hashimoto, S., Transactions of JSME, (in Japanese), Vol.68, No.669 (2002), pp.807-813. 
( 7 ) Lanes, L. and Laird, C., The Role of Annealing Twin Boundaries in the Cyclic Deformation of F.C.C. Materials, Mat. Sci. Eng., A, Vol.157 (1992), pp. 21-27.

( 8 ) Heintz, A. and Neumann, P., Crack Initiation during High Cycle Fatigue of an Austenitic Steel, Acta Metall., Vol.38, No.10 (1990), pp.1933-1940.

( 9 ) Boettner, R.C., McEvily Jr., A.J. and Liu, Y.C., On the Formation of Fatigue Cracks at Twin Boundaries, Phil. Mag., Vol.3 (1964), pp.95-106.

(10) Thompson, A.W., The Influence of Grain and Twin Boundaries in Fatigue Cracking, Acta. Metall., Vol.20 (1972), pp.1085-1094.

(11) Lim, L.C., Tay, Y.K. and Fong, H.S., Fatigue Damage and Crack Nucleation Mechanisms at Intermediate Strain Amplitudes, Acta Metall., Vol.38, No.4 (1990), pp.595-601.

(12) Yamashita, M., Mimaki, T., Hashimoto, S. and Miura, S., Stress Corrosion Cracking of [110] and [100] Tilt Boundaries in $\alpha$-Cu-Al Alloy, Phil. Mag., Vol.63 (1991), pp.707-726.

(13) Kargol, J.A. and Albright, D.L., The Effect of Relative
Crystal Orientation on the Liquid Metal Induced Grain Boundary Fracture of Aluminum Bicrystals, Metall. Trans. A, Vol.8 (1977), pp.27-34.

(14) Jin, N.Y. and Winter, A.T., Cyclic Deformation of Copper Single Crystals Oriented for Double Slip, Acta Metall., Vol.32, No.7 (1984), pp.989-995.

(15) Li, X.W., Wang, Z.G., Li, G.Y., Wu, S.D. and Li, X., Cyclic Stress-Strain Response and Surface Deformation Features of [011] Multiple-Slip-Oriented Copper Single Crystals, Acta Mater., Vol.46, No.13 (1998), pp.4497-4505.

(16) Iida, S., Ohno, K., Kamimae, H., Kumagai, H. and Sawada, S., Tables of Physical Constants, (1992), p.24.

(17) Obrtlik, K., Man, J. and Polak, J., Orientation Dependence of Surface Relief Topography in Fatigued Copper Single Crystals, Mater. Sci. Eng. A, Vol.234-236 (1997), pp.727-730.

(18) Melisova, D., Weiss, B. and Stickler, R., Nucleation of Persistent Slip Bands in Cu Single Crystals under Stress Controlled Cycling, Scri. Mater., Vol.36, No.9 (1997), pp.1061-1066. 